\title{
La Unión Bancaria (UB): nuevo marco competitivo para la banca española y europea*
}

\author{
The Banking Union (BU): New Competitive Framework \\ for Spanish and European Banks
}

\author{
Ángel Berges \\ Emilio Ontiveros \\ Catedráticos de la Universidad Autónoma de Madrid, \\ y Socios Fundadores de Afi, Analistas Financieros Internacionales
}

doi: 10.18543/ced-54-2016pp99-120

Sumario: I. Introducción.-II. Antecedentes: el rescate bancario en España como acelerador de la UB.-III. La UB: elementos principales.IV. El nuevo marco bancario europeo que define la UB.-V. A modo de recapitulación.

Resumen: El proyecto de la Unión Bancaria de Europa es el resultado de la respuesta de las autoridades europeas a la particularización en algunos sistemas bancarios de la eurozona de la crisis desencadenada en julio de 2007 en el sistema financiero estadounidense. La severidad del círculo vicioso entre el deterioro de los activos bancarios y la deuda pública de algunos países periféricos, particularmente intenso hasta el verano de 2012, obligó a paliar rápidamente una de las limitaciones originales de la Unión Monetaria. En este artículo se analiza la génesis del proyecto, su estructura - sus cuatro pilares fundamentales - y el sistema bancario resultante, con especial atención a la dinámica de concentración sectorial, intensificada adicionalmente como consecuencia de la mayor regulación bancaria.

Palabras claves: Unión Monetaria; Unión Bancaria; Concentración Bancaria.

Abstract: The project of a banking union in Europe is the response from European authorities to the particularization of the crisis, triggered in July 2007 in the financial system of the United States, in some banking systems of the euro area. The severity of the vicious circle created between the deterioration of bank assets and the public debt of some governments was particularly intense until the summer of 2012, forced to solve one of the original limitations of monetary union. This article discusses the genesis of the project, its structure - the four pillars- and the resulting system-banking, with special attention to the dynamics of sectoral concentration, more intensified as a result of increased bank regulation.

Keywords: European Monetary Union; Banking Union; Banking Concentration.

* Recibido el 20 de julio de 2015, aceptado el 27 de enero de 2016.

Cuadernos Europeos de Deusto 


\section{Introducción}

Europa nuevamente hizo de la necesidad virtud, al definir el horizonte de Unión Bancaria. La Unión Monetaria (UME) nació incompleta, sin el andamiaje institucional necesario para hacer frente a episodios de crisis como los provenientes de EEUU en el verano de 2007. Fue a medida que esa crisis se intensificaba cuando se echó en falta la existencia de una unión fiscal y de una unión bancaria. La definición del horizonte de esta última se formuló en plena crisis y, como comentaremos en las páginas que siguen, ha constituido una vía importante de fortalecimiento de la dinámica de integración.

Tras más de dos años de intensas negociaciones y desarrollos legislativos, y tras un año completo de exhaustivo ejercicio de evaluación de los principales bancos europeos, la Unión Bancaria (UB) iniciaba formalmente su andadura en noviembre de 2014. Concretamente el día 4 de ese mes, cuando el BCE (Banco Central Europeo) - más específicamente el MUS (Mecanismo Único de Supervisión), o SSM por sus siglas en inglés, adscrito al BCE - asumía las funciones de supervisión, siendo este uno de los dos pilares básicos que configuran la UB. El otro, el MUR (Mecanismo Único de Resolución) (SRM por sus siglas en inglés), entró en vigor el 1 de enero de 2016, si bien con un periodo transitorio de ocho años, en cuanto a la dotación del Fondo de Resolución.

Sin menoscabo de ese gradualismo en la plena operatividad del SRM, lo cierto es que la UB puede considerarse ya como una realidad plena, y la misma configura el nuevo marco competitivo relevante para la banca española y europea. En las notas que siguen se describen los antecedentes de la UB, sus pilares y mecanismos fundamentales de funcionamiento. Lo hacemos con referencias permanentes al sistema bancario español, ya sea desde la perspectiva de su contribución a ese proyecto o mediante el análisis de las consecuencias regulatorias y competitivas que esa integración bancaria va a significar sobre el conjunto del sistema.

\section{Antecedentes: El Rescate Bancario en España como acelerador de la UB}

Bien podría afirmarse que fue España, y más concretamente la crisis sufrida por su sistema bancario, uno de los principales aceleradores del proceso de construcción de la Unión Bancaria, como forma de romper con el peligroso círculo vicioso que se había desarrollado entre los riesgos bancarios y los riesgos asociados a la sostenibilidad de la deuda pública.

Ese círculo vicioso está implícito en lo que la literatura sobre economía financiera ha denominado «riesgo moral» («moral hazard») desde hace 
más de medio siglo. Ha sido objeto de numerosos análisis empíricos sobre las crisis bancarias: su magnitud, duración y efectos sobre la estabilidad y el crecimiento. Pero nunca antes los riesgos en una crisis bancaria habían sido de tal intensidad como para poner en peligro, con la generalidad con la que llegaron a hacerlo, la propia solvencia de los Estados, de tal manera que su capacidad para garantizar la estabilidad financiera se vio también cuestionada. Y, con ello, la capacidad de los sistemas bancarios para recuperar su normal funcionamiento, y garantizar el necesario flujo financiero hacia los sectores productivos.

Ese fue el caso en la eurozona, especialmente durante los años 2011 y 2012, cuando las dudas sobre la sostenibilidad de las finanzas públicas en algunos países, así como sobre la salud de algunos sistemas bancarios, desencadenaron un peligroso proceso de autoalimentación, un «bucle diabólico» en toda regla: las dudas sobre la deuda pública, en un marco de intensa contracción del crecimiento económico, se trasladaban a enormes dificultades de renovación de sus vencimientos por parte de los bancos, que respondían con fuertes restricciones en la concesión de crédito, que a su vez, agudizaban la recesión y la generación de ingresos públicos.

La contracción de la inversión crediticia iba a alimentar ese círculo vicioso entre el deterioro de los balances bancarios y el de las magnitudes macroeconómicas, a su vez ejerciendo una presión creciente sobre la vulnerabilidad de las cuentas públicas, y la consecuente debilidad sobre la sostenibilidad de la deuda pública. Esos cuestionamientos fueron especialmente intensos durante todo el año 2011, con fuertes incrementos en el coste diferencial de la deuda pública en los países periféricos - entre ellos España - y tensiones en los mercados primarios, con grandes dificultades para llevar a cabo las necesarias emisiones de deuda pública por los Tesoros.

El Banco Central Europeo (BCE) intentó resolver el problema de la financiación de los Estados, pero iba a generar una agudización del círculo vicioso entre riesgos bancarios y riesgos soberanos. Las inyecciones de financiación a tres años (conocidas como LTRO, «Long Term Financing Operations»), a un coste muy reducido (1\%, que posteriormente se reduciría al $0,5 \%$ ), y sin límite a la petición, siempre que las entidades bancarias contasen con el colateral suficiente, en deuda pública descontable. Los bancos españoles acudieron en masa a las dos subastas convocadas, en las que se adjudicaron más de 200.000 millones de euros. Como consecuencia de ello, el sistema bancario español alcanzó, en la primavera de 2012, una posición prestataria agregada superior a los 400.000 millones de euros, lo que representaba un $33 \%$ del préstamo total otorgado por el Eurosistema. Las cuantiosas compras de deuda pública que los bancos españoles llevaron a cabo con dicha financiación del BCE, iban a permitir a estos una cierta 
mejora en sus márgenes (gracias al denominado «carry trade», al percibir por la deuda pública mayores ingresos que el coste pagado al BCE), pero a cambio de intensificar todavía más el círculo vicioso entre riesgos bancarios y riesgo soberano.

De hecho, una actuación del BCE encaminada a facilitar financiación al sistema bancario, en un contexto de imposible financiación mayorista de los mismos, produciría, precisamente una consecuencia extraordinariamente perversa, cual es la de segmentar de una forma antes nunca vista la dependencia de los bancos respecto al BCE, como se aprecia en el gráfico 1.

Posición en TARGET2 de los bancos centrales nacionales del área euro

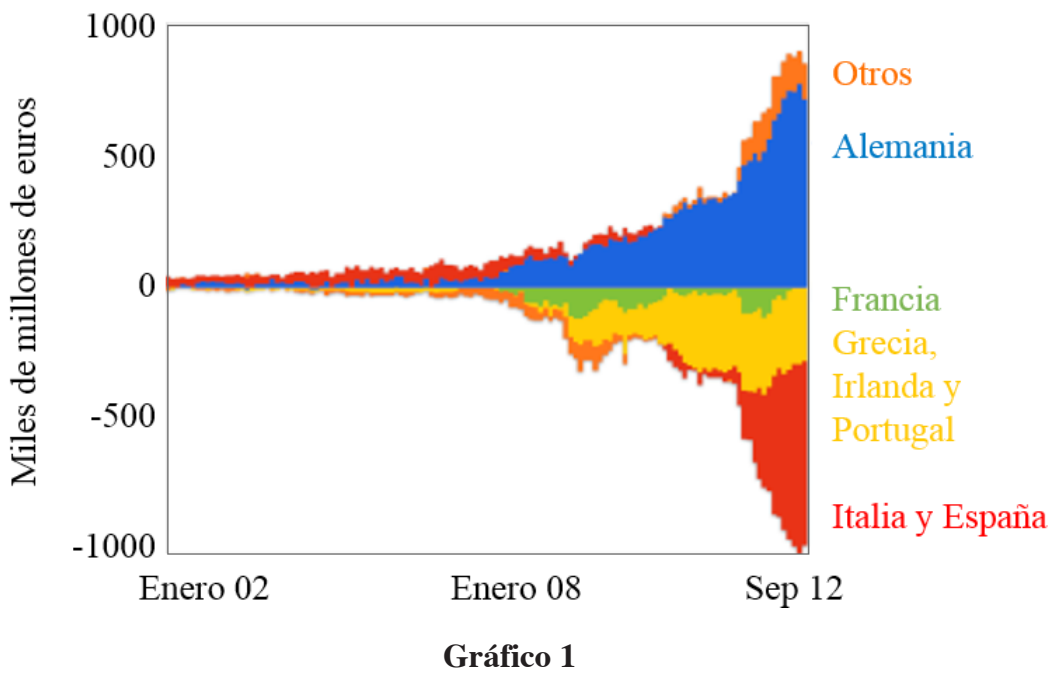

Máxima fragmentación en el Eurosistema

Fuente: FMI, Febrero SDN 13/01

Los bancos de las economías consideradas «periféricas» (España, Italia, Irlanda, Portugal y Grecia) pasaron a ser importantes deudores del BCE, a quien de forma indirecta convertían en el principal financiador de sus respectivos Tesoros, si bien el riesgo de crédito derivado de ese ascenso en los montantes de deuda pública permanecía en el sistema bancario de cada país. Por el contrario, los bancos de los países más saneados financieramente (Alemania, Holanda y Finlandia, fundamentalmente) asumían importantes posiciones acreedoras frente al Eurosistema. Esa asimetría definida 
por la posición de los sistemas bancarios frente al Eurosistema impedía la efectiva integración financiera en Europa. Esta alcanzaba su manifestación más preocupante durante los meses siguientes, cuando la percepción de fragmentación financiera alcanzó con toda su intensidad al corazón de los sistemas bancarios, es decir a su negocio minorista con clientes, de la que el gráfico 2 es suficientemente ilustrativo al respecto. El coste de los depósitos minoristas había sido históricamente muy homogéneo en toda Europa, con una variación que venía explicada fundamentalmente por la de los tipos de interés del BCE, que actuaban como referencia para todos los sistemas bancarios de la eurozona. Sin embargo, desde principios de 2010 esa homogeneidad se rompe, dando paso a una extraordinaria divergencia entre países, con una banda de fluctuación de casi 4 puntos porcentuales.
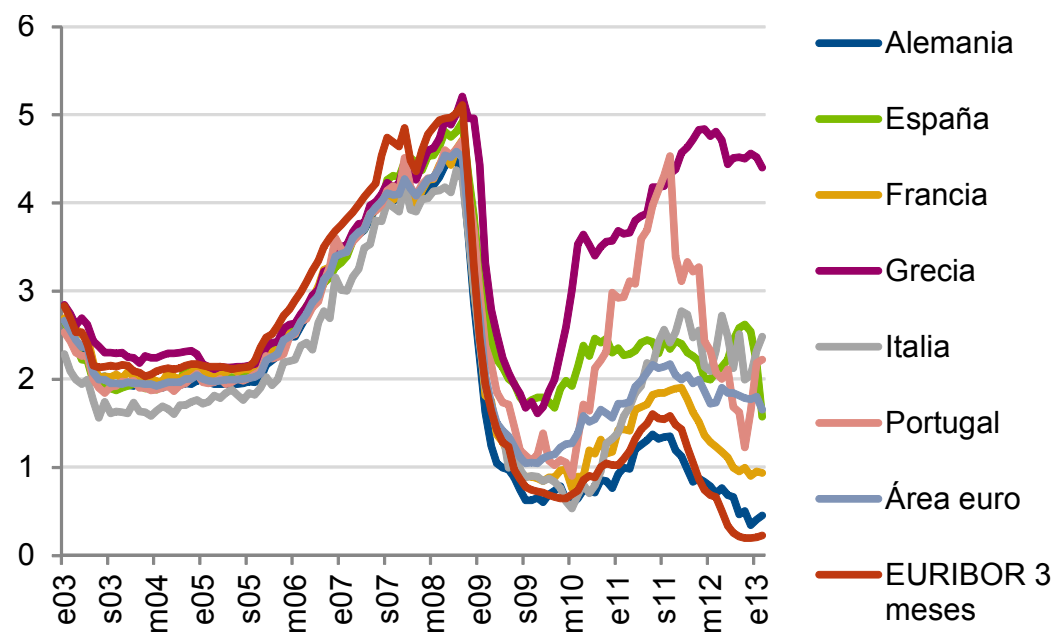

Gráfico 2

Máxima fragmentación en el negocio bancario minorista

(Coste de los depósitos bancarios por países, en \%)

Fuente: Afi basado en datos del BCE

Ese comportamiento asimétrico de los depósitos bancarios entre los diferentes bloques de países de la Eurozona pasó a caracterizar un sistema bancario absolutamente fragmentado y con evidentes riesgos para la estabilidad financiera del área monetaria. En el caso español alcanzaba su punto álgido en la primavera de 2012, con la intervención de Bankia, que dio lugar a una generalizada pérdida de confianza en el sistema bancario espa- 
ñol. El debilitamiento de la confianza tiene lugar en un momento en que, como hemos explicado anteriormente, la banca se había erigido en el principal - de hecho, casi en el único- comprador de las emisiones de deuda pública española, lo que incorporaba un peligroso factor de aceleración en el círculo vicioso entre riesgos bancarios y soberanos, al que hemos aludido en varias ocasiones.

La consecuencia fue extraordinariamente negativa para la percepción por los mercados de los dos riesgos, y para la capacidad de apelación a esos mercados para la renovación de vencimientos. La prima de riesgo española registraba fuertes incrementos, tanto la correspondiente al Tesoro como a los mayores bancos del sistema (únicos para los que existía un activo mercado secundario); de hecho, un elemento a destacar es la estrecha correlación existente entre las primas de riesgo bancario y soberano. Esa intensa asociación es sin duda el principal exponente del mecanismo autoalimentador entre los dos tipos de riesgos.

Pero más preocupante incluso que esa ampliación de las primas de riesgo en el mercado secundario, fue el colapso que se produjo en el mercado primario, o de emisiones, poniendo en extremo riesgo la posibilidad de hacer frente a los elevados vencimientos de deuda tanto por parte del Tesoro como de las entidades financieras. Fue esa percepción de riesgo extremo lo que abocó al gobierno a solicitar una línea de asistencia financiera (rescate) para su sistema bancario, que era aprobada por el Eurogrupo a finales de junio, por un importe máximo de 100.000 millones de euros. Se trataba del primer programa de rescate bancario, con las exigencias de cumplimiento de la condicionalidad estipulada en el Memorándum of Understanding, Mou: una intervención en toda regla sobre la capacidad de decisión de las autoridades españolas en materia que afectara al sistema bancario. La utilización de esa línea se llevaría a cabo por parte del FROB (Fondo de Reestructuración Ordenada Bancaria), quien a su vez inyectaría el capital directamente en las entidades necesitadas. Al ser el FROB una entidad pública, la deuda a asumir en el marco del «rescate bancario» se consolidaría como deuda pública. De esa cifra inicialmente aprobada como marco de recapitalización, poco más del 40\% (concretamente unos 41.400 millones de euros) han sido finalmente utilizados, unos 39.000 para capitalizar a las entidades necesitadas, y unos 2.400 para participar en el capital de la SAREB (Sociedad de Gestión de Activos Procedentes de la Reestructuración Bancaria), la sociedad creada para la gestión de los activos inmobiliarios transferidos desde las entidades capitalizadas con fondos públicos.

Las entidades que recibieron capital público se vieron obligadas, en el contexto del MoU descrito, a firmar unos acuerdos de condicionalidad («term sheets») en los que se comprometían a severos planes de reestructuración, fuerte reducción de capacidad (oficinas y empleados), así como ena- 
jenación de activos no vinculados al puro negocio minorista, y en general fuerte reducción de su base de crédito vivo. Ese fuerte descenso del crédito en las entidades más débiles, unido al estancamiento del mismo en el resto de entidades, se ha traducido en que el sistema bancario en términos agregados ha reducido en casi 400.000 millones (es decir un $40 \%$ del PIB) su saldo vivo de crédito a hogares y empresas no financieras, en un proceso de desapalancamiento que probablemente no ha finalizado.

En paralelo con esa reducción del crédito a hogares y empresas, los bancos españoles incrementaron de forma intensa su exposición al sector público, erigiéndose en los principales tenedores de deuda pública española (y de cuyas tenencias los bancos han obtenido jugosos márgenes, que en algunos casos han llegado a representar un $40 \%$ del margen total), acentuando el círculo vicioso entre riesgos bancarios y riesgos soberanos, cuya superación es uno de los objetivos de la Unión Bancaria que, tras intensos procesos negociadores, se pone en marcha a finales de 2014.

\section{La Unión Bancaria (UB): Mecanismos que la integran}

Aunque formalmente la Unión Bancaria (UB) constituye un paso más en la integración europea, y por tanto su ámbito «natural» de aplicación debería ser el conjunto de la Unión Europea, no cabe duda de que han sido las tensiones en el seno de la Eurozona - y muy especialmente ese mencionado círculo vicioso entre riesgos bancarios y riesgos soberanos que ha aquejado a varios países de la misma, entre ellos España- las que han actuado como principal revulsivo para el avance hacia la UB, por lo que su alcance principal se circunscribirá a los países de la Eurozona, estando en todo caso abierta al resto de países europeos.

En este sentido, y como ya hemos adelantado en la sección anterior, el rescate bancario español fue uno de los principales detonantes, o al menos «aceleradores» de la efectiva implantación de la UB. Concretamente, el hecho de que la capitalización de la banca se llevase a cabo a través del FROB, en lugar de hacerse directamente desde el MEDE (Mecanismo Europeo de Estabilidad). La capitalización a través del FROB mantiene el vínculo entre riesgos bancarios y soberanos, pues, en última instancia, si las entidades no son capaces de devolver el capital recibido, será el FROB quien asuma su devolución ante Europa. Si el origen del problema era la mencionada circularidad, o bucle diabólico, entre riesgos bancarios y soberanos, la forma más efectiva para romper dicho vínculo habría sido mediante una capitalización directa a los bancos desde Europa, sin pasar por el sector público español como garante de los riesgos bancarios. Sin embargo, la posición europea al respecto mantuvo en todo momento que ello solo se- 
ría objeto de consideración en el marco de una UB plenamente operativa, o al menos en el ámbito de una supervisión bancaria única, y suficientemente, implantada y experimentada.

El primer paso hacia esa unión bancaria se dio con la aprobación por el PE (Parlamento Europeo), en septiembre de 2013, del mecanismo único de supervisión (SSM, por sus siglas en inglés). Inició su andadura, bajo la responsabilidad del BCE - más específicamente, el Consejo de Supervisión, vinculado al $\mathrm{BCE}-$, a finales de 2014. Ese Consejo asume la supervisión directa de unas 130 entidades europeas - las que superan ciertos umbrales, en términos de tamaño absoluto y relativo, así como en términos de complejidad de su negocio - que representan en torno al $85 \%$ del total de activos bancarios totales de la Eurozona. Sobre el resto de entidades de menor dimensión y/o complejidad, unas 3.500, serán las autoridades supervisoras nacionales las que continúen ejerciendo esa función, si bien de forma estrechamente coordinada con el BCE (Consejo de Supervisión).

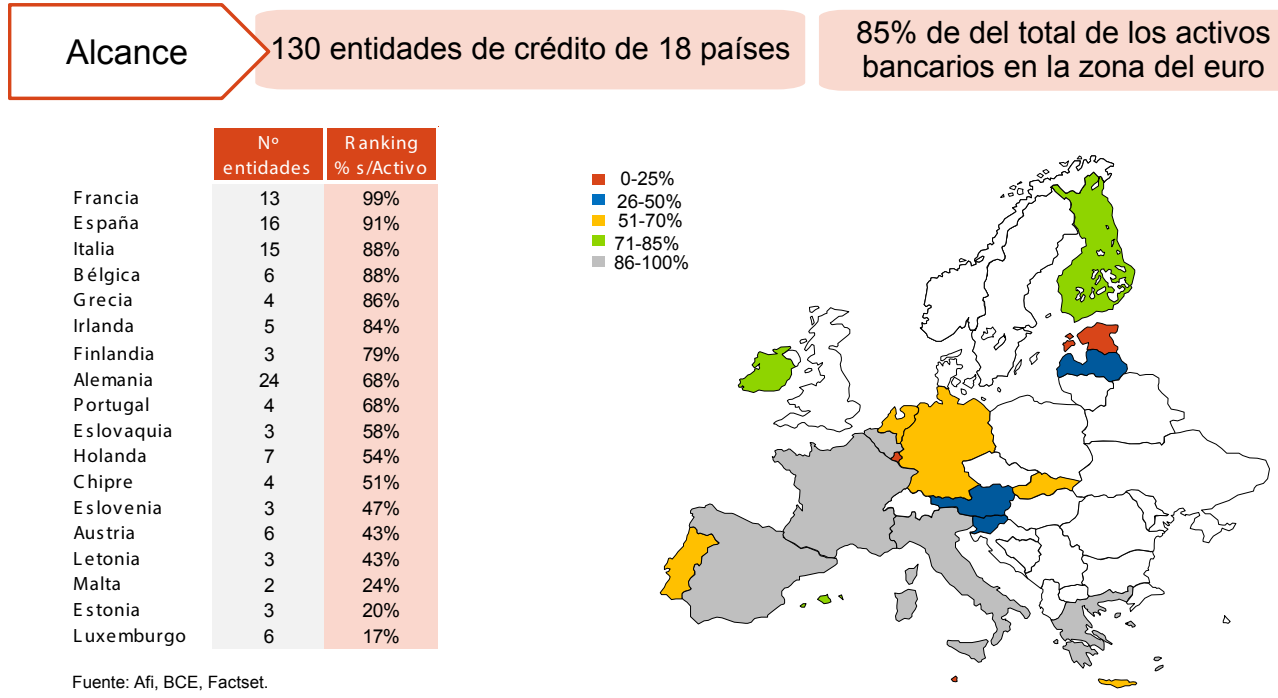

\section{Gráfico 3}

España es uno de los países con mayor peso de la supervisión directa, como puede apreciarse en el gráfico 3. A la fecha de entrada en vigor del MUS, un total de 16 entidades, representando el $91 \%$ de los activos totales del sistema bancario, pasaban a ser objeto de supervisión directa. El co- 
rolario es que tan sólo el 9\% restante de los activos (bien es cierto que gestionado por un elevado número de entidades de menor dimensión) quedaba bajo la supervisión directa del Banco de España, en todo caso a llevarse a cabo de forma coordinada con el MUS.

En este sentido, el gráfico 4 recoge la estructura organizativa del MUS, que descansa en cuatro Direcciones Generales (DG) que se encargan de la supervisión, asistidas por una Secretaría General.

Las DG de Supervisión Microprudencial I y II se encargan de la supervisión directa de las entidades de crédito significativas. Se organizan según un enfoque de supervisión basado en el riesgo y, por tanto, están especializadas según la exposición al riesgo, la complejidad y los modelos de negocio. La DG de Supervisión Microprudencial I se ocupa de los aproximadamente treinta grupos bancarios sistémicamente más importantes, mientras que la DG de Supervisión Microprudencial II lo hace de aproximadamente noventa grupos bancarios.

La supervisión de las entidades significativas se realiza en estrecha cooperación con las ANC (Autoridades Nacionales Competentes, que en el caso español es el Banco de España). Para cada entidad o grupo bancario significativo directamente supervisado por el BCE se ha establecido un equipo conjunto de supervisión (ECS), dirigido por un coordinador de las DG de Supervisión Microprudencial I y II del BCE, asistido por uno o más subcoordinadores locales de las ANC y formado por supervisores tanto del BCE como de las ANC de los países participantes. En aras de promover y subrayar la neutralidad, los coordinadores de ECS generalmente no provienen del país de origen de la entidad bajo su supervisión.

La DG de Supervisión Microprudencial III tiene asignada la función de vigilancia del $\mathrm{BCE}$ respecto de la supervisión directa de entidades menos significativas que realizan las ANC. El BCE puede asumir en cualquier momento, tras consultar con la ANC competentes o a solicitud de dicha ANC, la responsabilidad de supervisar directamente las entidades menos significativas para garantizar la aplicación coherente de normas de supervisión estrictas y la consistencia de los resultados de la supervisión.

Finalmente, la DG de Supervisión Microprudencial IV acoge todos los servicios horizontales y especializados de supervisión. Presta apoyo a los ECS y a las ANC en el ejercicio de la supervisión de entidades de crédito tanto significativas como menos significativas. Dada la amplia variedad existente hasta la fecha de marcos de supervisión nacionales (incluidas las políticas, metodologías, normas y procesos) y de prácticas, un objetivo fundamental de la DG de Supervisión Microprudencial IV es promover el desarrollo y la aplicación de un enfoque de supervisión único, coherente y avanzado en aras de la igualdad de condiciones y de una supervisión más eficaz en toda Europa. 


\begin{tabular}{|c|c|c|c|c|}
\hline \multicolumn{2}{|c|}{ Supervisión directa } & Supervisión indirecta & $\begin{array}{c}\text { Supervisión horizontal } \\
\text { y conocimientos }\end{array}$ & Secretaría \\
\hline $\begin{array}{c}\text { Supervisión } \\
\text { Microprudencial I * }\end{array}$ & $\begin{array}{c}\text { Supervisión } \\
\text { Microprudencial II * }\end{array}$ & $\begin{array}{c}\text { Supervisión } \\
\text { Microprudencial III * }\end{array}$ & $\begin{array}{c}\text { Supervisión } \\
\text { Microprudencial IV * }\end{array}$ & $\begin{array}{l}\text { Secretaría del } \\
\text { Consejo de } \\
\text { Supervisión * }\end{array}$ \\
\hline $\begin{array}{l}\text { Supervisión de } \\
\text { Entidades de Cré- } \\
\text { dito Significati- } \\
\text { vas I } \\
\text { Supervisión de } \\
\text { Entidades de Cré- } \\
\text { dito Significati- } \\
\text { vas II } \\
\text { Supervisión de } \\
\text { Entidades de Cré- } \\
\text { dito Significati- } \\
\text { vas III } \\
\text { Supervisión de } \\
\text { Entidades de Cré- } \\
\text { dito Significati- } \\
\text { vas IV } \\
\text { Supervisión de } \\
\text { Entidades de Cré- } \\
\text { dito Significati- } \\
\text { vas V } \\
\text { Supervisión de } \\
\text { Entidades de Cré- } \\
\text { dito Significati- } \\
\text { vas VI } \\
\text { Supervisión de } \\
\text { Entidades de Cré- } \\
\text { dito Significati- } \\
\text { vas VII }\end{array}$ & $\begin{array}{l}\text { Supervisión de } \\
\text { Entidades de Cré- } \\
\text { dito Significati- } \\
\text { vas VIII } \\
\text { Supervisión de } \\
\text { Entidades de Cré- } \\
\text { dito Significati- } \\
\text { vas IX } \\
\text { Supervisión de } \\
\text { Entidades de Cré- } \\
\text { dito Significati- } \\
\text { vas X } \\
\text { Supervisión de } \\
\text { Entidades de Cré- } \\
\text { dito Significati- } \\
\text { vas XI } \\
\text { Supervisión de } \\
\text { Entidades de Cré- } \\
\text { dito Significati- } \\
\text { vas XII } \\
\text { Supervisión de } \\
\text { Entidades de Cré- } \\
\text { dito Significati- } \\
\text { vas XIII } \\
\text { Supervisión de } \\
\text { Entidades de Cré- } \\
\text { dito Significati- } \\
\text { vas XIV } \\
\text { Supervisión de } \\
\text { Entidades de Cré- } \\
\text { dito Significati- } \\
\text { vas XV }\end{array}$ & $\begin{array}{l}\text { Apoyo Analítico y } \\
\text { Metodológico } \\
\text { Vigilancia Institu- } \\
\text { cional y Sectorial } \\
\text { Vigilancia Supervi- } \\
\text { sora y Relaciones } \\
\text { con ANC }\end{array}$ & $\begin{array}{l}\text { Análisis de Riesgos } \\
\text { del MUS } \\
\text { Autorizaciones } \\
\text { Control de Calidad } \\
\text { Supervisora } \\
\text { Ejecución y Sancio- } \\
\text { nes } \\
\text { Gestión de Crisis } \\
\text { Inspecciones in situ } \\
\text { Centralizadas } \\
\text { Desarrollo y meto- } \\
\text { dología y Normas } \\
\text { Modelos Internos } \\
\text { Políticas Supervi- } \\
\text { soras } \\
\text { Planificación y } \\
\text { Coordinación de } \\
\text { Programas de Exa- } \\
\text { men Supervisor }\end{array}$ & $\begin{array}{l}\text { Política de } \\
\text { adopción de } \\
\text { decisiones }\end{array}$ \\
\hline
\end{tabular}

\section{Gráfico 4}

Mecanismo único de Supervisión: Estructura organizativa

Este organigrana muestra los dos primeros niveles de la estructura organizativa de las nuevas áreas de actividad

\section{(*) DG (Dirección General)}


El segundo pilar constitutivo de la UB, que sin duda resultó mucho más difícil de consensuar entre los gobiernos europeos, se refiere al mecanismo único de resolución (MUR, o SRM por sus siglas en inglés). Tras arduas negociaciones en el seno de las instituciones europeas (Comisión Europea, Consejo Europeo, BCE, y Parlamento Europeo), se llegó finalmente a un consenso sobre su articulación. Fue aprobado por el Parlamento Europeo el 15 de abril de 2014, es decir en su última sesión plenaria previa a la disolución de la legislatura, lo cual era condición necesaria para la puesta en marcha del mismo en 2015, como pieza complementaria del SSM. Si este se traduce en que los bancos europeos están sometidos a una supervisión común, el MUR (SRM) pretende garantizar que en caso de dificultades todos los bancos europeos estarán sometidos a las mismas reglas de resolución, y sus diferentes acreedores serán tratados de forma homogénea, con independencia del país de origen. Se trata, en resumen, de garantizar la resolución ordenada de los bancos en dificultades, de una forma homogénea entre países, y bajo el principio de minimización del coste para los contribuyentes.

El MUR está compuesto por dos elementos separados, pero altamente complementarios entre sí: una Junta Única de Resolución (más conocida por sus siglas en inglés SRB, Single Resolution Board), y un Fondo Único de Resolución (FUR, o SRF, Single Resolution Fund).

La Junta de Resolución (SRB) se configura como el principal órgano de decisión del MUR, en la medida en que es quien decide sobre los sistemas de resolución a aplicar a aquellos bancos no viables - la no viabilidad de un banco será en general decidida por el supervisor (MUS), aunque también podría darse el caso de que fuese la propia Junta de Resolución quien decidiese - incluyendo la aplicación de instrumentos de resolución y, en su caso, el uso del Fondo de Resolución. Esas decisiones tomadas por la Junta de Resolución deberán, en todo caso, someterse a la Comisión Europea, que dispondrá de un plazo de doce horas para formular objeciones; en caso de no existir estas serán firmes las decisiones de la Junta.

Un mecanismo de resolución eficaz debe reducir al mínimo la posibilidad de que los costes de la resolución de una entidad inviable sean asumidos por los contribuyentes. Debe también garantizar que las entidades de envergadura sistémica sean objeto de resolución sin poner en peligro la estabilidad financiera. El instrumento de recapitalización interna (bail-in) persigue este objetivo garantizando que los accionistas y los acreedores de la entidad inviable sufran las pérdidas pertinentes y asuman la parte correspondiente de los costes que se deriven de la inviabilidad de la entidad. El instrumento de recapitalización interna supondrá por tanto un importante incentivo para que los accionistas y los acreedores de las entidades controlen la salud de una entidad en circunstancias normales, y cumple la recomendación de que en el marco de resolución se incluyan competencias es- 
tatutarias de reducción y conversión del valor de la deuda como una opción adicional junto con otros instrumentos de resolución.

En todo caso, el instrumento de recapitalización interna debe aplicarse de manera que se trate de forma similar a los acreedores y se respete el rango estatutario de las reclamaciones conforme a la legislación aplicable en casos de insolvencia. Las pérdidas deben ser absorbidas en primer lugar por los instrumentos de capital regulatorio y ser asignadas a los accionistas, bien mediante la cancelación o transmisión de acciones o a través de una fuerte dilución. Si estos instrumentos no son suficientes, debe procederse a una conversión o amortización de la deuda subordinada. Si las categorías subordinadas se han convertido o amortizado ya totalmente, debe procederse a una conversión de deuda «senior».

El segundo componente del MUR es el Fondo único de Resolución, que es un fondo establecido a nivel supranacional, financiado con las aportaciones de los bancos, y cuyo objetivo es actuar como mecanismo de capitalización de aquellos bancos en resolución, pero sólo tras haber agotado los mecanismos internos de recapitalización.

La definición de dicho Fondo de Resolución - en términos de monto total a alcanzar, perfil temporal de las aportaciones a realizar por los bancos, y ritmo en el grado de mutualización entre países - fue sin duda el principal elemento de negociación entre los gobiernos de la eurozona, cuyo consenso se alcanzó casi al final del límite temporal para su aprobación por el Parlamento Europeo en la anterior legislatura.

El acuerdo alcanzado supone que el FUR se constituirá de forma gradual durante un periodo de ocho años, al término del cual deberá alcanzar un importe dotado de al menos el 1\% de los depósitos con cobertura de todas las entidades de crédito autorizadas en todos los países que forman parte de la UB. De acuerdo con las cifras actuales de dichos depósitos, se estima que al término de los ocho años (enero de 2023) el Fondo deberá haber alcanzado un patrimonio de unos 55.000 millones de euros.

El Fondo estará compuesto inicialmente por «compartimentos nacionales». Estos se fusionarán progresivamente a lo largo de la fase transitoria de ocho años. Esta «mutualización» del uso de fondos aportados comenzará con el $40 \%$ en el primer año, el $20 \%$ en el segundo y aumentará constantemente en cantidades iguales a lo largo de los seis años restantes hasta que los compartimentos nacionales dejen de existir.

Es precisamente ese gradualismo en las aportaciones y en la mutualización de las mismas, así como el reducido importe final que el Fondo aspira a tener -55.000 millones para toda la UB, cuando sólo el rescate bancario en España requirió una cantidad superior a dicha cifra - lo que impide afirmar que la UB está ya plenamente operativa. En todo caso, la irreversibilidad de las decisiones tomadas - apoyadas en directivas, y en un Acuerdo 
Intergubernamental - otorga una credibilidad al proyecto de la UB cuyos efectos positivos deben notarse ya desde el primer momento de su puesta en marcha.

\section{EI Nuevo Marco Bancario Europeo}

La efectiva entrada en funcionamiento de la UB, aún con ese régimen transitorio para las dotaciones y mutualización de los fondos de resolución, representa un paso irreversible hacia un sistema bancario europeo integrado, que configura un nuevo marco competitivo en el que las entidades españolas llevarán a cabo su negocio desde el momento mismo de alumbramiento de la mencionada UB.

En principio, tan sólo los sistemas bancarios de la Eurozona estarán sometidos a la supervisión única, por lo que podría pensarse que la misma delimita el ámbito geográfico e institucional relevante. Ahora bien, en la medida en que la Unión Bancaria es una construcción en el marco de la más amplia Unión Europea, y todos los países integrantes de la misma tienen la opción de solicitar su acceso a la supervisión común, entendemos que el ámbito competitivo relevante es el de la UE en su totalidad.

El sistema bancario europeo así definido (UE) se erige, sin duda alguna y con gran diferencia, en el mayor del mundo, con casi 40 billones de euros de activos totales gestionados ( 28 de los cuales corresponden a países de la Eurozona), frente a los aproximadamente 12 billones que representa el sistema bancario de Estados Unidos, o los 6 billones en el caso del sistema bancario japonés.

Ese elevado volumen de activos bancarios en Europa es indicativo de un grado de bancarización mucho más elevado en el continente europeo que en esas otras dos grandes potencias económicas. Concretamente, los activos bancarios expresados como porcentaje del PIB superan el 300\% en el caso europeo, mientras que en Estados Unidos apenas alcanzan un 100\%, y en Japón quedan en una posición intermedia, con un 150\%. La contrapartida a ese alto grado de bancarización en Europa frente a Estados Unidos es que en este último país tienen un peso mucho más importante los mercados de capitales en la financiación de las actividades productivas. De esta manera, Europa y Estados Unidos serían los dos extremos más representativos de lo que en la literatura sobre sistemas financieros comparados se ha definido como «banking oriented» (Europa), versus «market oriented» (Estados Unidos). Esa caracterización del sistema financiero europeo, especialmente el continental, como claramente «banking oriented», le otorga una especial sensibilidad al funcionamiento del sistema bancario en el desarrollo económico, mucho mayor que en el caso estadounidense. 
En todo caso, dentro del sistema bancario europeo tiene lugar una considerable heterogeneidad entre países en cuanto a bancarización, tanto relativa a PIB como a población, como se pone de manifiesto en el cuadro 1 adjunto. Merece destacarse que el grado de bancarización en España es muy similar a la media europea, en términos de negocio por habitante, pero algo superior si es referido al PIB. En este caso destaca que el crédito bancario en España representa 1,4 veces el PIB, frente a 1,1 veces en la media europea. De hecho España aparece en este epígrafe en unos niveles similares a los de Irlanda, Portugal o Reino Unido, y claramente superados por los de Holanda o Dinamarca.

\section{Cuadro 1}

Sistemas bancarios europeos: Indicadores de bancarización (2014)

\begin{tabular}{|c|c|c|c|c|c|c|c|c|c|c|}
\hline & \multicolumn{3}{|c|}{ Veces sobre PIB } & \multicolumn{3}{|c|}{ Miles $€$ / habitante } & \multicolumn{3}{|c|}{ Millones $€ / \mathrm{km}^{2}$} & \multirow{2}{*}{$\begin{array}{c}\text { Millones } € \\
\text { Volumen de } \\
\text { negocio / } \\
\text { Empleado }\end{array}$} \\
\hline & Activo & Crédito & Depósitos & Activo & Crédito & Depósitos & Activo & Crédito & Depósitos & \\
\hline Alemania & 2.5 & 0.9 & 1.2 & 83.6 & 31.1 & 38.7 & 18.9 & 7.0 & 8.7 & 8.6 \\
\hline Austria & 3.5 & 1.1 & 1.1 & 128.9 & 40.2 & 38.8 & 13.0 & 4.1 & 3.9 & 8.8 \\
\hline Bélgica & 2.6 & 0.8 & 1.3 & 86.0 & 26.9 & 45.3 & 31.5 & 9.8 & 16.5 & 13.8 \\
\hline Dinamarca & 3.5 & 2.0 & 0.7 & 155.4 & 87.2 & 29.9 & 20.2 & 11.3 & 3.9 & 18.1 \\
\hline España & 3.4 & 1.4 & 1.5 & 74.6 & 30.9 & 32.0 & 6.9 & 2.9 & 3.0 & 13.6 \\
\hline Francia & 3.1 & 1.1 & 1.0 & 97.1 & 33.2 & 30.5 & 9.9 & 3.4 & 3.1 & 10.0 \\
\hline Grecia & 1.9 & 1.1 & 0.9 & 33.4 & 19.7 & 15.4 & 2.8 & 1.6 & 1.3 & 7.6 \\
\hline Holanda & 4.1 & 1.7 & 1.5 & 145.0 & 62.2 & 53.0 & 58.6 & 25.1 & 21.4 & 20.0 \\
\hline Irlanda & 4.8 & 1.4 & 1.3 & 172.0 & 50.0 & 45.0 & 11.2 & 3.3 & 2.9 & 12.3 \\
\hline Italia & 1.7 & 1.1 & 1.0 & 44.1 & 28.2 & 25.6 & 8.7 & 5.6 & 5.1 & 10.5 \\
\hline Portugal & 2.8 & 1.4 & 1.3 & 44.0 & 22.4 & 20.3 & 5.0 & 2.6 & 2.3 & 7.8 \\
\hline Reino Unido & 4.8 & 1.4 & 1.5 & 145.4 & 43.7 & 44.8 & 38.2 & 11.5 & 11.8 & 13.4 \\
\hline Total UE & 3.0 & 1.1 & 1.3 & 78.1 & 28.9 & 33.2 & 8.9 & 3.3 & 3.8 & 10.2 \\
\hline
\end{tabular}

Fuente: Afi, BCE, Eurostat.

Si el análisis se hace relativizando por persona, en vez de PIB, la conclusión es diferente, pues el crédito bancario por habitante es en España algo menor que en la media europea; de hecho, tan sólo Italia, Portugal y Grecia presentan niveles de crédito per cápita inferiores al español. Algo similar se concluye cuando el análisis se realiza en relación a la superficie (en kilómetros cuadrados), un tipo de análisis que es bastante común realizar en estudios sobre 
distribución espacial. En este epígrafe, y en paralelo con una densidad demográfica en España claramente inferior a la media europea, también la conclusión es que el grado de bancarización en España es muy similar a la media europea.

De ese diagnóstico, que claramente apunta a una saturación relativa de los sistemas bancarios en Europa, e incluso más en España, cabe concluir que el nuevo entorno bancario en el sistema europeo integrado va a ser incluso más competitivo y con menor margen de crecimiento, lo que sin duda incorporará presión adicional sobre las entidades para la optimización de su gestión.

Ello nos lleva a abordar el más relevante elemento de contraste entre los sistemas bancarios europeos, cual es el referido a la estructura empresarial de los mismos, y el grado de rivalidad competitiva que de esa estructura emerge.

Acorde con un elevado grado de bancarización en prácticamente todos los países europeos, situación que no es nueva sino que viene de bastantes décadas atrás, los sistemas bancarios de la mayoría de países se caracterizan por una elevada presencia de entidades, en muchos casos con un ámbito territorial de actuación bastante reducido. Esa estructura empresarial -así como la referida a la red de oficinas, que abordaremos más adelante - tiene también unas raíces históricas vinculadas a una visión tradicional del negocio bancario en el que la cercanía - física, pero también personal - tenía una gran importancia en la prestación de servicios por las entidades bancarias. Sin poner en duda que esos valores puedan seguir teniendo relevancia, lo cierto es que su peso relativo va siendo cada vez menor, frente a la generalización de nuevos canales de distribución y/o de relación con los clientes bancarios, así como frente a las exigencias de mayor racionalidad empresarial, en un contexto de saturación del negocio.

La confluencia de todos los efectos anteriores ha provocado una reducción del censo de entidades en la totalidad de países europeos, como ilustra el cuadro 2 adjunto. Esa reducción, medida sobre el periodo entre 2008 y 2013 (los años más afectados por la crisis bancaria), alcanza casi un 10\% en el agregado europeo de tal manera que, tras esa disminución, el agregado de sistemas bancarios en la UE contaba con un censo de entidades, al cierre de 2013, de 7.726, una cifra sin duda demasiado elevada, y que con toda probabilidad será objeto de reducciones adicionales conforme cobre cuerpo la plena integración europea a nivel de negocio bancario minorista.

Del citado cuadro puede deducirse cómo la reducción en el censo ha sido generalizada en la totalidad de países, si bien con diferente intensidad. En este sentido, España es, tras Grecia, el país cuyo sistema bancario ha registrado una mayor reducción porcentual en su censo, de un $28 \%$, a lo largo de la crisis. 


\section{Cuadro 2}

Sistemas bancarios en Europa: Censo de entidades y concentración

\begin{tabular}{lrrr|rc} 
& \multicolumn{3}{c|}{ Número de entidades } & \multicolumn{2}{c}{ Concentración (\%) } \\
\cline { 2 - 6 } & 2008 & 2013 & Variación & 2008 & 2014 \\
\hline Alemania & 1.882 & 1.734 & $-7,9 \%$ & 23 & 32 \\
Austria & 771 & 701 & $-9,1 \%$ & 39 & 37 \\
Bélgica & 49 & 39 & $-20,4 \%$ & 81 & 66 \\
España & 282 & 204 & $-27,7 \%$ & 42 & 58 \\
Francia & 672 & 579 & $-13,8 \%$ & 51 & 48 \\
Grecia & 36 & 21 & $-41,7 \%$ & 70 & 94 \\
Holanda & 266 & 204 & $-23,3 \%$ & 87 & 85 \\
Irlanda & 472 & 431 & $-8,7 \%$ & 55 & 48 \\
Italia & 729 & 611 & $-16,2 \%$ & 31 & 41 \\
Portugal & 147 & 127 & $-13,6 \%$ & 69 & 69 \\
\hline Total UE & 8.525 & 7.726 & $-9,4 \%$ & 44 & 47 \\
\hline
\end{tabular}

(*) Cuota de activos de las 5 entidades de mayor tamaño del país (\%)

Fuente: Afi, sobre datos de BCE (2014) «Banking Structures Report».

Una implicación directa de esa reducción del censo ha sido el incremento en el grado de concentración, medido por la cuota que representan las cinco mayores entidades en cada país, incremento que ha tenido lugar en la mayoría de países, aunque hay algunas excepciones, como Francia, Holanda, Bélgica e Italia. España es el país europeo en el que el grado de concentración ha experimentado un incremento más acusado, pasando de un $42 \%$ a un $58 \%$, entre 2008 y 2014.

El grado de concentración así medido no puede ser objeto de comparación indiscriminada entre países, pues depende del tamaño y profundidad de cada país. Así puede comprobarse cómo la cuota de las cinco mayores entidades es mucho más elevada en los países pequeños (donde el número de entidades es menor) que en los grandes. Con dicho matiz en mente, merece destacarse que, entre los cuatro grandes países de la Eurozona, España es el que presenta actualmente un mayor grado de concentración, medido por la cuota de las cinco mayores entidades. Dicha cuota es en España del $58 \%$, frente a $48 \%$ en Francia, $41 \%$ en Italia, y $32 \%$ en Alemania, siendo este último país el que presenta un mayor grado de atomización, con más de 1.700 entidades, es decir más que entre Francia, Italia y España juntas. Sin duda alguna la existencia de numerosas entidades de ámbito regional, o in- 
cluso local, en dicho país, está detrás de una configuración empresarial tan atomizada.

Por otra parte, ante la gradual, pero irreversible, integración bancaria europea, los indicadores convencionales de concentración a nivel de cada país perderán gradualmente virtualidad. Frente a ellos habrá que incorporar un marco de medición dual, que tome en consideración el ámbito de negocio relevante para cada entidad. De esta manera las entidades con vocación global deberán tomar en consideración la concentración a nivel europeo (actualmente las cinco mayores entidades europeas representarían algo menos del $20 \%$ de cuota agregada sobre el conjunto del sistema bancario), mientras que para entidades con un ámbito de actuación más acotado territorialmente será menos relevante esa cuota a nivel europeo, siéndolo mucho más la cuota en sus mercados locales de actuación.

Finalmente, merece la pena resaltar que, pese a la tendencia generalizada a la reducción del censo, se siguen creando nuevas entidades que tratan de abrirse un hueco en nichos de mercado prometedores, fundamentalmente en el ámbito de los nuevos canales de distribución y la apuesta por la banca digital.

Esa mención a los canales de distribución nos lleva al otro gran aspecto que conforma la estructura de los sistemas bancarios europeos, cual es el descansar en una extensa red de oficinas físicas, con amplia dotación de empleados, como medio principal de distribución de los servicios bancarios, así como de relación con los clientes y usuarios de dichos servicios.

Como puede comprobarse en el cuadro 3, al cierre de 2014 y para el sistema bancario europeo en conjunto, su capacidad de producción y/o distribución de servicios descansaba en una dotación total de 204.000 oficinas, y un total de 2,9 millones de empleados, ámbitos los dos (oficinas y empleados) en los que también se ha producido una importante reducción desde el inicio de la crisis, al igual que ocurrió con el número de entidades. La disminución ha sido del 14\% en número de oficinas, y del 11\% en el número de empleados.

España es uno de los países donde esa contracción ha sido más intensa, del $30 \%$ en número de oficinas, y del $27 \%$ en número de empleados. Ese mayor esfuerzo de reducción de capacidad en nuestro país tiene una doble justificación. Por un lado el hecho de ser uno de los países en los que el impacto de la crisis ha sido mayor, y con ello el del saneamiento con ayudas públicas, lo cual tiene obvias implicaciones, en términos de exigencia de ajuste de capacidad, en el marco de la normativa europea sobre ayudas de Estado. Esta argumentación sería válida sobre todo para explicar la reducción de empleados. 


\section{Cuadro 3}

Sistemas bancarios europeos: Indicadores de ajuste de capacidad

\begin{tabular}{lrrr|rrr} 
& \multicolumn{3}{c}{ Número de oficinas } & \multicolumn{3}{c}{ Número de empleados } \\
\cline { 2 - 7 } & 2008 & 2014 & Variación & \multicolumn{1}{c}{2008} & 2014 & Variación \\
\hline Alemania & 39.531 & 35.284 & $-10,7 \%$ & 685.550 & 647.300 & $-5,6 \%$ \\
Austria & 4.243 & 4.247 & $-0,1 \%$ & 78.754 & 74.110 & $-5,9 \%$ \\
Bélgica & 4.316 & 3.607 & $-16,4 \%$ & 65.985 & 56.611 & $-14,2 \%$ \\
Dinamarca & 2.192 & 1.186 & $-45,9 \%$ & 52.830 & 37.201 & $-29,6 \%$ \\
España & 46.065 & 31.999 & $-30,5 \%$ & 276.497 & 201.643 & $-27,1 \%$ \\
Francia & 39.467 & 37.623 & $-4,7 \%$ & 424.536 & 411.012 & $-3,2 \%$ \\
Grecia & 4.098 & 2.688 & $-34,4 \%$ & 66.163 & 45.654 & $-31,0 \%$ \\
Holanda & 3.421 & 1.854 & $-45,8 \%$ & 116.000 & 94.000 & $-19,0 \%$ \\
Irlanda & 895 & 994 & $11,1 \%$ & 40.507 & 28.871 & $-28,7 \%$ \\
Italia & 34.169 & 30.723 & $-10,1 \%$ & 338.035 & 299.684 & $-11,3 \%$ \\
Portugal & 6.417 & 5.938 & $-7,5 \%$ & 62.377 & 53.888 & $-13,6 \%$ \\
Reino Unido & 12.270 & 11.381 & $-7,2 \%$ & 491.262 & 402.561 & $-18,1 \%$ \\
\hline Total UE & 237.647 & 204.146 & $-14,1 \%$ & 3.259 .306 & 2.889 .320 & $-11,4 \%$ \\
\hline
\end{tabular}

Fuente: Afi, sobre datos de BCE.

En cuanto a oficinas, junto a la anterior existe otra clara justificación, y es una especialización tradicional del modelo de oficinas en España en torno a una excesiva capilaridad, buscando la cercanía física a los clientes, mediante una estrategia de apertura de numerosas oficinas bancarias de tamaño muy reducido. Esa especialización en muchas oficinas pequeñas puede extraerse del cuadro anterior si se constata cómo, en 2008, el sistema bancario español contaba con 46.000 oficinas bancarias, es decir el $20 \%$ del total europeo, cuando en volumen de activos bancarios, o en PIB, España apenas alcanzaba el 9\% o 10\% del total europeo.

Esa especialización en una red de distribución muy granular (numerosas oficinas pequeñas) se pone de manifiesto en el gráfico 5 , en el que se representan los principales sistemas bancarios europeos (los seis mayores, que representan casi el $80 \%$ del total de activos) en una doble dimensión en cuanto a su estructura de distribución: capilaridad de la red (oficinas por cada millón de habitantes), y tamaño medio de cada oficina (empleados por oficina). A su vez, para cada uno de esos países se presenta la situación existente antes de la crisis (2008) junto a la actual, para ilustrar la dirección en la que está teniendo lugar el desplazamiento. 


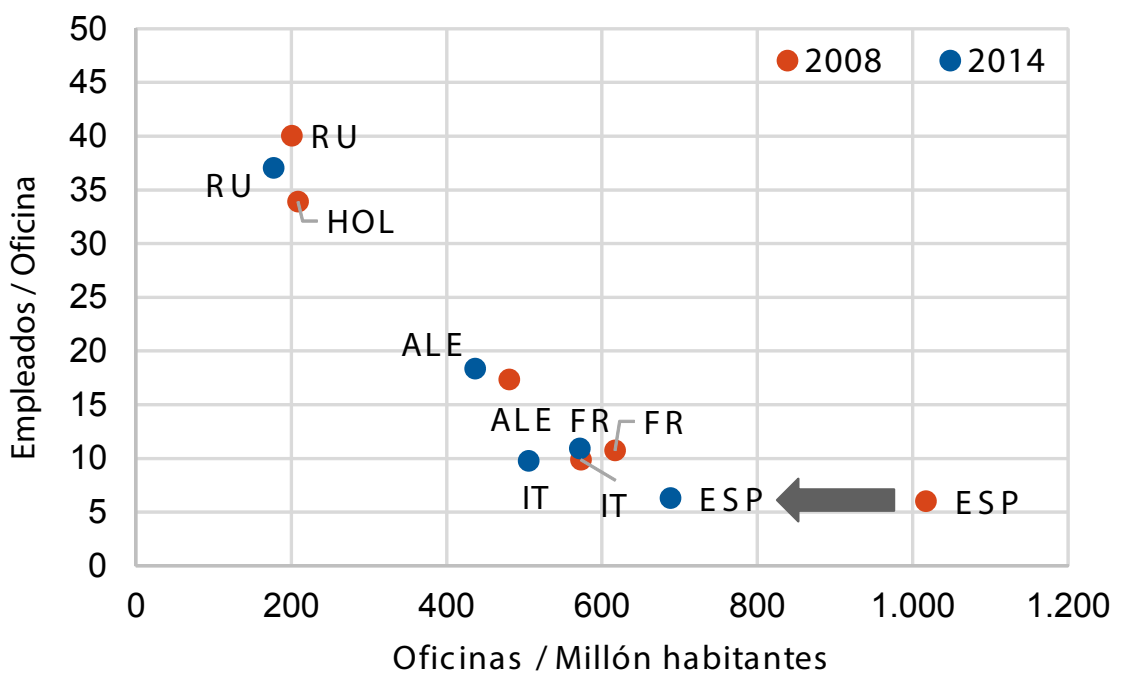

\section{Gráfico 5}

Sistemas bancarios europeos: Densidad versus tamaño medio de oficinas

Fuente: Afi, BCE, Eurostat.

De dicho gráfico se desprenden claramente dos modelos de distribución. Por un lado los de Holanda y Reino Unido, con una especialización en oficinas grandes (en torno a 40 empleados por oficina), pero con muy escasa capilaridad (200 oficinas por cada millón de habitantes, equivalente a una oficina por cada 5.000 habitantes). Y por otro los de Alemania, Francia e Italia, con oficinas pequeñas (entre 10 y 15 empleados) y mucho más capilares (500 o 600 oficinas por cada millón de habitantes). El caso extremo de ese modelo es el español, con el menor tamaño medio de oficina (5 o 6 empleados) y la máxima capilaridad, si bien esta se ha reducido algo a consecuencia del cierre de oficinas registrado desde el inicio de la crisis. Dicha capilaridad ha pasado de más de 1.000 oficinas por cada millón de habitantes, a unas 700 en la actualidad, ratio que sigue siendo el de mayor capilaridad en Europa (y tal vez en el mundo), lo que apunta a que el proceso de cierre de oficinas en España todavía no ha terminado.

Un último aspecto a matizar es el referido a esa capilaridad en relación con la densidad demográfica. Probablemente no sea casual que los dos países (Holanda y Reino Unido) con menor capilaridad son precisamente aquellos en los que la densidad poblacional es más elevada. En una configuración demográfica muy concentrada, como es el caso de esos dos países, 
cobra mucha menos relevancia el disponer de una red de oficinas con alta capilaridad, algo que sí que puede ser relevante en países muy extensos, y con muy baja densidad demográfica, como es el caso de España.

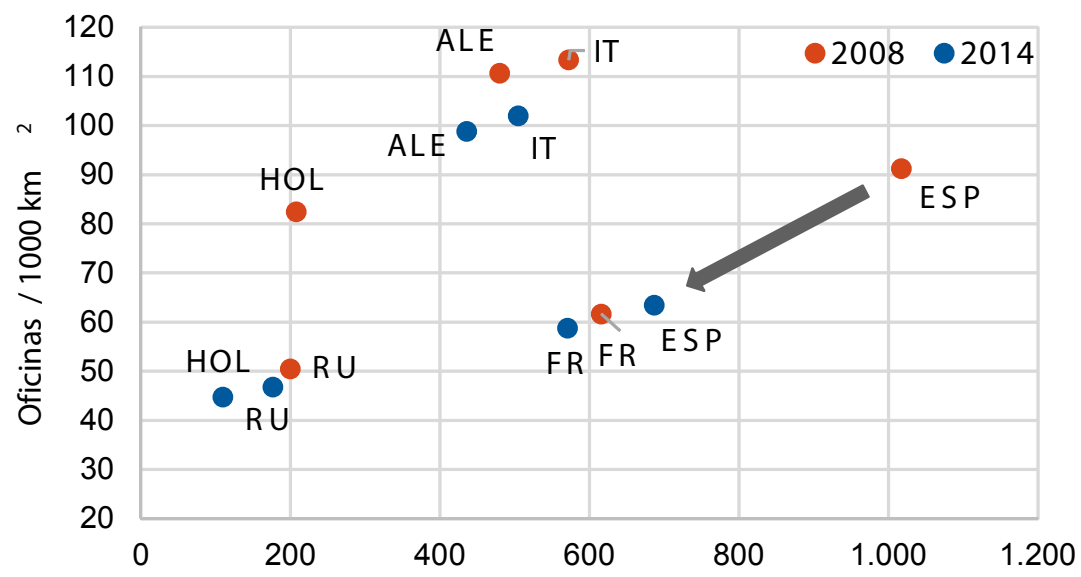

Oficinas / Millón habitantes

\section{Gráfico 6}

Sistemas bancarios europeos:

Demografía y densidad de la red de oficinas

Fuente: Afi, BCE, Eurostat.

En este sentido, el gráfico 6 representa la red de oficinas en los principales sistemas bancarios, pero incorporando la dimensión espacial, medida en términos de oficinas por cada 1000 kilómetros cuadrados. Puede comprobarse cómo en este caso el sistema bancario español converge mucho más hacia los de países como Reino Unido, Holanda y Francia, mientras que Alemania o Italia mantienen una red más tupida, relativa a la superficie del país.

Esa comparación espacial puede haber sido relevante en el pasado, pero sin duda tiene escasa virtualidad ante el futuro. Una muy densa red de oficinas en España, asociada a una muy reducida densidad demográfica, y a una estrategia de acercamiento físico de las oficinas a los clientes bancarios, pudo ser válido en un modelo de negocio en el que los clientes visitaban las oficinas, que se erigían en el principal canal de prestación de servicio y de comunicación con los usuarios de servicios financieros. Pero las tecnologías 
de la información y de la comunicación, y, sobre todo, los nuevos hábitos de los consumidores de servicios financieros hace que ese valor de las oficinas como nexo de unión con los clientes se vea fuertemente erosionado, en cuyo caso todo apunta a que el proceso de racionalización de la red de oficinas en España está lejos de haber concluido.

En todo caso, teniendo en cuenta la mencionada especialización diferencial en un modelo de muchas oficinas pequeñas, no debe concluirse que España necesite un ajuste más intenso que los principales países europeos, al menos en cuanto a dotación de personas dedicadas al negocio de intermediación financiera. Retomando los datos del cuadro 3 anterior, un total de 200.000 empleados configuran la plantilla total de las entidades de crédito en España, tras haberse reducido dicha cifra en 75.000 desde 2008. A modo de contraste, en Italia son 300.000 empleados (es decir 1,5 veces España), en Francia son más de 400.000 (dos veces España), y en Alemania 650.000 (3,2 veces España). Bien, pues a modo de contraste de dichas cifras relativas, cabe recordar que, ni en población, ni en PIB, ni en activos bancarios productivos, supera Italia a España en 1,5 veces, ni Francia en 2 veces, ni Alemania en 3,2 veces. De ahí se deriva que la productividad del empleo en el sistema bancario español es claramente superior al de sus homólogos italiano, francés o alemán. $\mathrm{O}$, alternativamente, si se asume que el sistema bancario español debe realizar recortes adicionales de capacidad (en términos de empleo), esa necesidad de recorte sería mucho más imperiosa en los otros tres grandes países de la Eurozona.

\section{A modo de recapitulación}

La Unión Bancaria (UB) ha fortalecido la dinámica de integración europea y, en todo caso, ha fortalecido las tareas de supervisión y resolución de crisis bancarias en la eurozona. Era de todo punto necesaria en la región más bancarizada del mundo, en la que la dependencia bancaria de la financiación empresarial era prácticamente total. Ello se explica porque el colapso del crédito bancario durante la crisis supuso una restricción financiera sin precedentes en varios países europeos, entre ellos el nuestro. Esa situación sólo ha podido ser paliada cuando el Banco Central Europeo (BCE) ha desplegado todo su arsenal de medidas encaminadas a estimular la predisposición de los bancos a canalizar financiación a empresas y familias. Junto a esa proactividad del BCE, la propia puesta en marcha de la UB ha incorporado un factor adicional de normalización de la actividad bancaria, en la medida en que un nuevo y más creíble marco de supervisión y resolución añade solidez al sistema crediticio, facilitando con ello el normal restablecimiento de las actividades de intermediación. 
Pero más allá de esa «normalización» de la condiciones bancarias que la UB ha traído consigo, el sistema financiero europeo continúa adoleciendo de una insuficiente diversificación de fuentes de financiación, y exhibiendo una excesiva dependencia de la financiación bancaria, especialmente cuando se le compara con el sistema financiero de Estados Unidos, país que sufrió mucho menos las consecuencias del colapso bancario, al disponer de fuentes alternativas de financiación mucho más desarrolladas.

Precisamente en esa dirección, de favorecer el desarrollo de nuevas fuentes de financiación al margen del sistema bancario, va encaminada la otra gran iniciativa puesta en marcha por la Unión Europea (UE) como extensión natural de la Unión Bancaria. Se trata de la Unión de los Mercados de Capitales que, a diferencia de la UB - limitada a los países de la Eurozona, al menos en sus inicios - pretende integrar desde el primer momento a todos los países de la UE. Esta iniciativa procura, además de reducir los riesgos de «sobrebancarización», garantizar una mayor adecuación de la estructura institucional y de la oferta de instrumentos de financiación a la desigual composición de la demanda según los riesgos asociados a la misma. La conjunción y normal funcionamiento de ambos permitirá hablar de un verdadero espacio financiero europeo.

\section{Bibliografía}

AFI, Guía del Sistema Financiero Español, 7. a edición (en prensa, edición prevista septiembre 2015).

BANCO CENTRAL EUROPEO, Financial Stability Review, mayo 2015.

BERGES, A., ONTIVEROS, E., «Austerity and financial instability», The Economic Crisis and Governance in the European Union, Routledge Studies in the European Economy, 2014, pp. 155-181.

BERGES, A., ONTIVEROS E., VALERO F.J., «La hoja de ruta del sistema bancario español ante la unión bancaria europea», Papeles de Economía Española, n. ${ }^{\circ} 137,2013$, pp. 122-136.

INTERNATIONAL MONETARY FUND, «A banking union for the euro area», IMF Staff Discussion Note, febrero 2013.

SHAMBAUGH, J.C., «The euro's three crisis», Brookings Papers on economic activity, marzo 2012. 


\section{Derechos de autor (Copyright)}

Los derechos de autor de esta publicación pertenecen a la editorial Universidad de Deusto. El acceso al contenido digital de cualquier número de Cuadernos Europeos de Deusto (CED) es gratuito, transcurridos 6 meses desde su publicación. Los trabajos podrán descargarse, copiar y difundir, sin fines comerciales y según lo previsto por la ley. Así mismo, los trabajos editados en CED pueden ser publicados con posterioridad en otros medios o revistas, siempre que el autor indique con claridad y en la primera nota a pie de página que el trabajo se publicó por primera vez en CED, con indicación del número, año, páginas y DOI (si procede). 